\title{
Avaliação dos parâmetros hematológicos e bioquímicos de ratas no segundo terço da gestação submetidas à ação do extrato metanólico de Cereus jamacaru DC., Cactaceae
}

\author{
Júlio B. Messias, ${ }^{* 1}$ Mércia C. M. Caraciolo, ${ }^{2}$ Inalda M. de Oliveira, ${ }^{3}$ Ulisses $R$. Montarroyos, ${ }^{4}$ \\ Isla V. G. A. Bastos, ${ }^{5}$ Martha de O. Guerra, ${ }^{6}$ Ivone A. Souza ${ }^{5}$
}

${ }^{l}$ Instituto de Ciências Biológicas, Universidade de Pernambuco, Rua Arnóbio Marques, 310, Santo Amaro, 52210-220 Recife-PE, Brasil,

${ }^{2}$ Centro de Pesquisas Aggeu Magalhães, Fiocruz, Av. Professor Moraes Rego s/n, Cidade Universitária, 50670-420 Recife-PE, Brasil,

${ }^{3}$ Centro Acadêmico da Vitória de Santo Antão, Universidade Federal de Pernambuco, Rua Alto do Reservatório s/n, Bela Vista, 55608-680 Vitória de Santo Antão-PE, Brasil,

${ }^{4}$ Faculdade Maurício de Nassau, Rua Fernando Lopes, 778, Graças, 52011-220 Recife-PE, Brasil,

${ }^{5}$ Departamento de Antibióticos, Universidade Federal de Pernambuco, Av. Prof. Moraes Rego s/n, Cidade Universitária, 50670-901 Recife-PE, Brasil,

${ }^{6}$ Centro de Biologia da Reprodução, Universidade Federal de Juiz de Fora, Caixa Postal 328, 36001-970

Juiz de Fora-MG, Brasil.

\begin{abstract}
RESUMO: Os efeitos da administração oral do extrato metanólico (EM) de Cereus jamacaru DC., Cactaceae, foram investigados sobre os parâmetros hematológicos e bioquímicos em ratas Wistar adultas grávidas. Vinte ratas ( $\mathrm{n}=5$ por grupo) foram tratadas durante quatro dias consecutivos com EM por via oral nas doses de 100, 250 e $500 \mathrm{mg} / \mathrm{kg}$ de peso e, em seguida, determinados os perfis bioquímico e hematológico. Os resultados mostraram que durante o período do tratamento não foi observado efeitos nocivos ou óbitos. Os parâmetros hematológicos e bioquímicos não foram modificados pela administração oral do EM, excetuando-se o aumento significativo de 45,7\% e de $41,9 \%$ para alanina transferase (ALT) nas doses de 250 e $500 \mathrm{mg} / \mathrm{kg}$ além do aumento significativo, respectivo de 48,2\%, 39,8\% e 41,8\% para aspartato aminotransferase (AST). Em relação aos valores hematológicos, registrou-se flutuação dentro dos valores de referência na contagem diferencial de hemoglobina, de neutrófilo e de linfócito. Dessa forma, a administração do extrato metanólico de C. jamacaru não apresentou reações tóxicas sobre a maioria dos parâmetros hematológicos e bioquímicos estudados em ratas Wistar adultas grávidas. Entretanto, o aumento dos níveis séricos de AST e ALT em doses elevadas sugere uma sobrecargas hepática, as quais devem ser investigadas em maiores detalhes.
\end{abstract}

Unitermos: Cereus jamacaru, mandacaru, prenhez, hematologia, bioquímica.

\begin{abstract}
Evaluation hematological and biochemical parameters of rats in the second thirds of the gestation submitted to the action of the extract methanol of Cereus jamacaru DC., Cactaceae". The effects of oral administration of methanol extract (ME) prepared from stems of Cereus jamacaru DC., Cactaceae were investigated on the biochemical and hematological parameters in pregnant adult Wistar rats. Twenty rats $(n=5$ per group) have been treated orally for four consecutive days with ME in doses of 100,250 and $500 \mathrm{mg} / \mathrm{kg}$ weight, and then, it was determined the biochemical and hematological profiles. The results showed that during the period of treatment there was no signs of toxicity or death. The hematological and biochemical parameters were not modified by oral administration of ME, except for a significant increase of $45.7 \%$ and $41.9 \%$ for alanine transaminase (ALT) in doses of 250 and $500 \mathrm{mg} / \mathrm{kg}$ in addition to the significant increase, of to $48.2 \%, 39.8 \%$ and $41.8 \%$ for aspartate transaminase (AST). In hematology, it was registered a fluctuation within the reference values of hemoglobin in the differential count of neutrophil and lymphocyte. In this way the administration of methanol extract of $C$. jamacaru does not produce toxic effects or alters the majority of biochemical and hematological studies in pregnant adult Wistar rats. However, the increase of serum ALT and AST in high doses suggests a liver overload, which must be investigated in more detail.
\end{abstract}

Keywords: Cereus jamacaru, mandacaru, pregnancy, hematology, biochemistry. 


\section{INTRODUÇÃO}

Cereus jamacaru DC., Cactaceae, é conhecido popularmente como mandacaru, mandacaru-de-boi, mandacaru-facheiro, mandacaru-de-faixo, cardeiro, jamacaru, jamaracurú, jumucurú, jumarucú, cumbeba e urumbeba (Zappi \& Aona, 2007).

É extremamente rústico, cresce nas catingueiras arbóreas e em locais quase desprovidos de solos, e se multiplica regularmente, cobrindo extensas áreas da caatinga (Lima, 1996), sendo um vegetal comum em praticamente todo o nordeste brasileiro, ocorrendo nos estados da Bahia, Maranhão, Pernambuco, Sergipe e também em Minas Gerais (Zappi \& Aona, 2007).

O mandacaru é utilizado principalmente na alimentação de ruminantes nos longos períodos de seca que ocorrem na região nordeste (Cavalcanti \& Resende, 2006). Os seus frutos servem de alimentos para pássaros e animais silvestres da caatinga (Cavalcanti \& Resende, 2007), e que apesar de insípido são bastante apreciados pela população (Barbosa, 1998), sendo inclusive uma alternativa para a fabricação de vinho (Almeida et al., 2006). A sua polpa, de acordo com Pimentel Gomes (2007) é doce e comestível, e a semelhança de outras cactáceas pode ser utilizada na alimentação humana (Salema, 1966; Albuquerque \& Andrade, 2002; Silva et al., 2005a).

A raiz do mandacaru, sob a forma de infuso ou decocto, é utilizada no tratamento de distúrbios renais, digestivos, respiratórios e hepáticos (Agra et al., 2007). As cascas do caule raspadas e curtidas em água, também são utilizadas para distúrbios renais (Albuquerque \& Andrade, 2002) e no controle do colesterol alto (Magalhães, 2006), enquanto a polpa do caule, misturada ao açúcar, é usada no tratamento de úlceras estomáquicas (Agra et al., 2007). Outras indicações populares para o uso da casca e do caule do mandacaru são para o combate à sífilis, e doenças vertebrais (Andrade et al., 2006a, b), como laxativo (Motta, 2003), como antiescorbútico, vermífugo, cicatrizante, antitumoral de origem glandular, cardiotônico (Correa, 1969), febrífugo (Lima, 1996) e como antiinflamatório (Gomes, 1972).

No estudo da toxicidade aguda foi verificado que o extrato bruto de $C$. jamacaru não apresentou ação cumulativa; em doses menores produziu efeito estimulante no sistema nervoso central, enquanto doses mais elevadas apresentaram efeitos depressores (Souza et al., 2000). O extrato hidroetanólico de Cereus jamacaru apresentou inibição tumoral evidente sobre tumores induzidos em camundongos (Sarcoma 180), contudo, se faz necessários estudos farmacológicos mais aprofundados para avaliar a potencialidade desse vegetal (Souza et al., 2001). Silveira et al. (2006) verificaram que em concentrações de 25,50 e $100 \mathrm{mg} / \mathrm{kg}$ o extrato etanólico de C. jamacaru não provocou nenhuma alteração hematológica quando administrado nos quatro primeiros dias da gestação.

De acordo com Davet et al. (2009) os componentes principais de C. jamacaru são duas aminas (tiramina e a $\mathrm{N}$-metiltiramina), sendo estas consideradas pelos autores, marcadores da espécie. Além dessas aminas os autores citam ainda a presença da hordenina e tirosina. A tiramina é o primeiro alcalóide derivado da tirosina, sendo formada por descarboxilação simples (Mann, 1987), enquanto a hordenina é resultante da metilação da tiramina. Todas essas aminas são alcalóides encontrados em diversos alimentos como abacate, laranja, banana, repolho (Vieira, 2006).

A tiramina, assim como as aminas a ela relacionadas age de forma indireta, modificando o acúmulo e a liberação de norepinefrina nas terminações nervosas. Estas aminas permitem que a norepinefrina interaja com seus receptores, porque retiram o neurotransmissor das áreas de reserva nas vesículas sinápticas ou de locais de ligação extravesicular (Bruton et al., 2006).

É curioso a falta de estudos que avaliem os possíveis efeitos deste vegetal no desenvolvimento do concepto, uma vez que trata-se de uma planta com toxidade indefinida e de uso comum na população brasileira, tanto na medicina dita popular, quanto na alimentação humana e animal. Portanto, o objetivo desse trabalho foi avaliar a toxicidade e alguns parâmetros bioquímicos séricos e hematológicos de ratas grávidas sob ação de diferentes doses do extrato metanólico de Cereus jamacaru DC., Cactaceae, no período da organogênese.

\section{MATERIAL E MÉTODOS}

\section{Material botânico}

Para a realização do estudo, foram utilizados exemplares de Cereus jamacaru DC., Cactaceae, coletados no município de Igarassú-PE, em março de 2007. O material botânico foi depositado no Herbário Geraldo Mariz do Departamento de Botânica, Centro de Ciências Biológicas da Universidade Federal de Pernambuco UFPE, sob registro 43010.

O extrato de $C$. jamacaru foi obtido através de maceração da parte interna do caule. E cuja obtenção, os exemplares foram lavados, cortados, e macerados em álcool metílico PA (Vetec $\left.{ }^{\circledR}\right)$ em um liquidificador industrial. Após este processo de maceração, material vegetal e o solvente foram mantidos ao abrigo da luz durante quatorze dias. Em seguida o material foi filtrado e concentrado em rotaevaporador para eliminação do solvente orgânico $\left(45^{\circ} \mathrm{C}\right)$ para obtenção do extrato metanólico bruto.

\section{Animais}

Foram utilizadas ratas Wistar, Rattus norvegicus var. albinus com peso de $+280 \mathrm{~g}$, provenientes do Biotério do Departamento de Antibióticos, Universidade Federal de Pernambuco. Os animais receberam água e dieta $\left(\right.$ Labina $\left.^{\circledR}\right)$ ad libitum e foram mantidos sob condições controladas de 
iluminação (ciclo $12 \mathrm{~h}$ claro/escuro) e temperatura $(23 \pm 2$ $\left.{ }^{\circ} \mathrm{C}\right)$. O protocolo experimental foi aprovado pelo Comitê de Ética em Experimentação Animal (CEEA) da Universidade Federal de Pernambuco (Processo n²3076.018858/200727).

Vinte fêmeas correspondendo a quatro grupos ( $\mathrm{n}=5$ por grupo), foram tratadas durante quatro dias consecutivos, com administração por via oral (gavage), do extrato metanólico de C. jamacaru nas doses de 100, 250 e $500 \mathrm{mg} / \mathrm{kg}$ (grupos tratados) e soro fisiológico (grupo controle), durante o terço médio da gestação, ou seja, os animais receberam as doses nos dias 8, 9, 10 e 11 após a fertilização. Durante a gestação todas foram avaliadas de acordo com os sinais clínicos de toxicidade e tiveram a sua massa corpórea registrada. No $15^{\circ}$ dia foram anestesiadas com uma mistura de ketamina $(85 \mathrm{mg} / \mathrm{kg}$ ) e xilazina ( $3 \mathrm{mg}$ / $\mathrm{kg}$ ), de acordo com Ferro (2007), para a coleta do sangue por punção cardíaca.

O sangue foi acondicionado em dois tipos de tubo: um com anticoagulante (EDTA) BD (Vacutainer ${ }^{\circledR}$ ) para determinação dos parâmetros hematológicos, e o outro, sem anticoagulante BD (Vacutainer ${ }^{\circledR}$ ) para avaliação dos parâmetros bioquímicos (Silva et al., 2005b).

\section{Avaliação da toxicidade}

A toxicidade foi avaliada através de observação dos sinais clínicos nos animais (abortamento, diarréia, piloereção, taquicardia, perda de peso e óbito), após a administração do extrato metanólico durante o período do tratamento.

\section{Análise bioquímica}

Os parâmetros glucose, uréia, creatinina, aspartato aminotransferase (AST), alanina aminotransferase (AST), colesterol total, triacilglicerídeos, fosfatase alcalina, bilirrubinas (total e direta), proteína total e frações (albumina e globulina) foram obtidos no aparelho analisador bioquímico Modular Analytic P (Roche ${ }^{\circledR}$ ) com sistemas comerciais do mesmo fabricante (Silva et al., 2005b).

\section{Análise hematológica}

Os valores para os eritrócitos, a hemoglobina, o hematócrito, os índices hematimétricos, os leucócitos (contagem total, relativa e absoluta) e as plaquetas foram determinados segundo Silva et al. (2005b) utilizando o analisador automático Sysmex XT 1800i $\left(\right.$ Roche $^{\circledR}$ ). A contagem diferencial de leucócitos foi realizada em extensões coradas com Panótico Rápido InstantProv $\left(\right.$ Newprov $\left.^{\circledR}\right)$. Em cada ensaio, 100 células foram analisadas e contadas (Silva et al., 2005b).

\section{Análise estatística}

Os valores foram expressos como média \pm erro padrão da média. As diferenças entre os grupos foram determinadas através da Análise de Variância (ANOVA oneway), seguida, quando detectada diferença, pelo teste de Bonferroni. O nível de significância para rejeição da hipótese de nulidade foi sempre $\geq$ a $5 \%$.

\section{RESULTADOS}

Durante o tratamento, não foram observados sinais clínicos de toxicidade e nenhum óbito foi registrado. O tratamento durante a fase da gestação estudada de forma geral, não induziu modificações no perfil bioquímico, uma vez que os parâmetros mantiveram-se dentro da faixa de referência (Harkness \& Wagner, 1993; Cubas et al.,, 2007), como para os valores observados no grupo controle. A exceção foi observada em relação a ALT nos grupos tratados com as duas doses mais elevadas, os quais aumentaram significantemente em 45,7 e 41,9\% ( $p<0,05, \mathrm{n}$ $=5$ ) assim como, a AST que aumentaram, respectivamente, em 48,2, 39,8 e 41,8\% $(p<0,05, \mathrm{n}=5)$, nos diferentes grupos tratados em relação ao grupo controle (Tabela 1).

Ao analisar os parâmetros hematológicos observou-se que a administração por via oral nos animais tratados com o extrato metanólico não apresentou alteração significativa (Tabela 2), embora flutuações na contagem diferencial de hemoglobina, de neutrófilos e de linfócitos tenham sido registrados.

\section{DISCUSSÃO}

A avaliação hematológica representa uma importante área de estudo sobre o estado de saúde dos animais. O hemograma e a análise bioquímica do sangue podem auxiliar o diagnóstico e o prognóstico de diversas enfermidades (Swenson, 1988; Jain, 1993). Os resultados mostraram que a administração oral durante quatro dias do extrato metanólico de $C$. jamacaru não produziu reações comportamentais em ratas Wistar adultas gestantes, uma vez que durante o tratamento, nenhum sinal clínico visível de toxicidade foi observado.

O perfil hematológico e bioquímico das fêmeas gestantes esteve dentro dos valores de referência (Harkness \& Wagner, 1993; Cubas et al., 2007), havendo, contudo, exceções para a AST e ALT.

As alterações na concentração das enzimas aminotransferases séricas (ALT e AST) e da fosfatase alcalina (FA) são importantes indicadores de lesões nas células hepáticas (Motta, 2003; Milinkovic-tur et al., 2005) e de acordo com Moncorvo et al. (1998) a dosagem da concentração das enzimas ALT, AST, FA e da Gama Glutamil Transferase (GGT) permitem averiguar a presença de alterações da permeabilidade dos hepatócitos pela elevação de ALT e AST, assim como de desordens 
colestáticas pela elevação de FA e GGT. A amplitude do aumento da concentração de uma determinada enzima em particular, geralmente, está correlacionada ao número de células hepáticas afetadas. A lesão do hepatócito, normalmente está associada a certo grau de colestase, independentemente das possíveis causas (inflamatória, degenerativa ou neoplásica), uma vez que os canalículos biliares podem ficam obstruídos em conseqüência da dilatação das células hepáticas (Moncorvo et al., 1998).

O aumento nos níveis plasmáticos de ALT e AST observados neste estudo foi estatisticamente diferente em relação ao grupo controle (Tabela 1) e divergiu tanto dos valores citados por Cubas et al. (2007), quanto dos apresentados por Dantas et al. (2006), neste a AST e a ALT variaram entre 69,3 a $92,7 \mathrm{U} / \mathrm{L}$ e 38,7 a $63,3 \mathrm{U} / \mathrm{L}$, respectivamente. Segundo Motta (2003) estes dados fornecem indícios de alterações na função hepática, uma vez que a AST e ALT são enzimas presentes em altas concentrações no músculo, fígado e cérebro, e a elevação da sua atividade no sangue pode indicar necrose ou moléstia especialmente nesses tecidos. AALT é encontrada principalmente no citoplasma do hepatócito, enquanto que $80 \%$ da AST encontra-se presente na mitocôndria. Assim as alterações nos valores séricos dessas enzimas auxiliam

Tabela 1. Efeito do extrato metanólico de Cereus jamacaru DC (100, 250 e $500 \mathrm{mg} / \mathrm{kg})$, administrados por via oral sobre os parâmetros bioquímicos em ratas Wistar adultas, tratadas no terço médio da gestação

\begin{tabular}{lcccccc}
\hline \multicolumn{1}{c}{ Parâmetros } & Normalidade 1 & Normalidade 2 & Controle & EE $100 \mathrm{mg} / \mathrm{kg}$ & EE 250 mg/kg & EE 500 mg/kg \\
\hline Glucose (mg/dL) & $50.0-135.0$ & $50.0-135.0$ & $127.4+12.13$ & $130.8+5.16$ & $131.4+19.97$ & $130.2+22.56$ \\
Uréia (mg/dL) & $15.0-21.0$ & $15.0-54.0$ & $45.8+9.47$ & $40.4+27.89$ & $51+10.67$ & $47.6+13.31$ \\
Creatinina (mg/dL) & $0.2-0.8$ & $0.2-0.8$ & $0.42+0.10$ & $0.47+0.05$ & $0.47+0.04$ & $0.51+0.04$ \\
Ast (U/L) & ---- & $39.0-92.0$ & $93.7+4.92$ & $138.84+5.74 *$ & $130.96+6.32 *$ & $132,86+9.44 *$ \\
Alt (U/L) & ---- & $17.0-50.0$ & $49.9+5.0$ & $60.94+5.25$ & $72.7+6.26^{*}$ & $70.8+8.77 *$ \\
Colesterol total (mg/dL) & $40.0-130.0$ & $40.0-130.0$ & $70.8+15.67$ & $83.8+15.06$ & $81.6+13.25$ & $77.6+18.90$ \\
Triacilglicerídeos (mg/dL) & $26.0-145.0$ & $26.0-145.0$ & $78.8+13.4$ & $91.4+7.43$ & $89.4+5.72$ & $90.2+5.40$ \\
Fosfatase alcalina (U/L) & ---- & $39.0-216.0$ & $120.2+19.84$ & $124.6+7.16$ & $104.6+11.28$ & $114.6+10.14$ \\
Bilirrubina total (mg/dL) & $0.20-0.55$ & $0.20-0.55$ & $0.30+0.05$ & $0.39+0.06$ & $0.34+0.07$ & $0.25+0.30$ \\
Proteínas totais (g/dL) & $5.6-7,6$ & $5.6-7.6$ & $6.5+0.54$ & $6.17+0.41$ & $6.41+0.95$ & $6.50+0.64$ \\
Albumina (g/dL) & $3.8-4.8$ & $3,3-4.9$ & $4.1+0.35$ & $4.00+0.31$ & $3.9+0.59$ & $3.94+0.40$ \\
Globulina (g/dL) & $1.8-3.0$ & $1.3-3.9$ & $2.9+0.27$ & $2.14+0.15$ & $2.45+0.46$ & $2.59+0.51$ \\
\hline
\end{tabular}

Os valores representam a média \pm e.p.m. de cinco dosagens obtidos em analisador bioquímico Modular Analytic P (Roche ${ }^{\circledR}$ ) com sistemas comerciais do mesmo fabricante. Normalidade 1: Harkness \& Wagner, 1993; Normalidade 2: Cubas et al., 2007; *Estatisticamente diferente do grupo controle (Teste ANOVA - oneway) seguido de Teste de Bonferroni, $p<0,05$ ).

Tabela 2. Efeito do extrato metanólico de Cereus jamacaru DC (100, 250 e $500 \mathrm{mg} / \mathrm{kg})$, administrados por via oral sobre os parâmetros hematológicos em ratas Wistar adultas, tratadas no terço médio da gestação.

\begin{tabular}{lcccccc}
\hline \multicolumn{1}{c}{ Parâmetros } & Normalidade 1 & Normalidade 2 & Controle & EE $100 \mathrm{mg} / \mathrm{kg}$ & EE 250 mg/kg & EE 500 mg/kg \\
\hline Eritrócitos $\left(10^{6} / \mu \mathrm{L}\right)$ & $7.0-10.0$ & $5.4-10.0$ & $6.47+0.57$ & $6.26+0.40$ & $5.89+0.53$ & $5.88+0.24$ \\
Hemoglobina $(\mathrm{g} / \mathrm{dL})$ & $11.0-18.0$ & $11.0-18.0$ & $12.5+0.89$ & $12.32+0.38$ & $11.46+0.61^{*}$ & $11.44+0.30^{*}$ \\
Hematócrito $(\%)$ & $36.0-48.0$ & $35.0-49.0$ & $39.18+3.43$ & $38.92+1.67$ & $36.5+2.63$ & $37.88+0.40$ \\
VCM (fL) & $48.0-51.4$ & $49-64$ & $60.58+1.80$ & $62.2+0.64$ & $62.02+1.48$ & $65.56+3.04$ \\
HCM (pg) & $15.7-18.0$ & $18-20.4$ & $19.34+0.74$ & $19.52+0.46$ & $19.5+0.81$ & $19.5+0.71$ \\
CHCM (g/dL) & $30.6-37.5$ & $31.4-36.6$ & $31.93+0.96$ & $31.4+0.46$ & $31.44+0.82$ & $30.2+1.01$ \\
Leucócitos $\left(10^{3} / \mu \mathrm{L}\right)$ & $6.0-17.0$ & $4.0-17.0$ & $4.63+1.03$ & $4.23+0.14$ & $4.20+0.22$ & $4.40+0.74$ \\
Neutrófilos $(\%)$ & $9.0-34.0$ & $9.0-50.0$ & $24.6+8.38$ & $26.8+3.83$ & $33.4+0.89 *$ & $31+1.87$ \\
Eosinófilos $(\%)$ & $0-6.0$ & $0-6.0$ & $1.0+0.71$ & $0.8+0.45$ & $1.0+0.71$ & $0.6+0.55$ \\
Basófilos $(\%)$ & $0-1.5$ & $0-1.5$ & $0.2+0.45$ & $0.2+0.45$ & $0.4+0.55$ & $0.2+0.45$ \\
Linfócitos $(\%)$ & $65.0-85.0$ & $50.0-85.0$ & $70.4+8.29$ & $69.4+5.32$ & $60.2+4.08^{*}$ & $65.0+3.53$ \\
Monócitos $(\%)$ & $0-5.0$ & $0-5.0$ & $3.8+2.77$ & $2.8+2.68$ & $5.0+3.45$ & $3.2+2.58$ \\
Plaquetas $(103 / \mu \mathrm{L})$ & $5.0-13.0$ & $4.5-8.85$ & $7.43+2.64$ & $6.78+0.48$ & $6.80+0.26$ & $7.63+0.97$ \\
\hline Os & & & & & &
\end{tabular}

Os valores representam a média \pm e.p.m. de cinco dosagens obtidos em analisador automático Sysmex XT 1800i (Roche $\left.{ }^{\circledR}\right)$. Normalidade 1: Harkness \& Wagner, 1993; Normalidade 2: Cubas et. al., 2007. VCM: Volume Corpuscular Médio, HCM: Hemoglobina Corpuscular Média, CHCM: Concentração de Hemoglobina Corpuscular Média. A contagem diferencial dos leucócitos foi realizada sobre extensões sanguíneas coradas com Panótico Rápido $\left(\mathrm{Newprov}^{\circledR}\right)$, onde 100 células foram analisadas para cada experimento *Estatisticamente diferente do grupo controle (Teste ANOVA - oneway) seguido de Teste de Bonferroni, $p<0,05$ ). 
no diagnóstico e no prognóstico de doenças hepáticas. Em danos hepatocelulares leves a forma predominante no soro é a citoplasmática, enquanto que em lesões graves há liberação da enzima mitocondrial, elevando a relação AST/ALT (Motta, 2003). De acordo com Moncorvo et al. (1998) os níveis aumentados da ALT é relativamente específica da doença hepatobiliar, enquanto o aumento dos níveis de AST em valores de dez vezes acima do limite superior de variação normal refletem normalmente uma patologia hepática ou biliar. Dessa forma os valores das aminotransferases são úteis para monitorizar a evolução da hepatopatia parenquimal aguda ou crônica. $\mathrm{O}$ aumento nos níveis de AST e ALT nos animais tratados com as diferentes doses $(100,250$ e $500 \mathrm{mg} / \mathrm{kg})$ pode indicar um aumento na hepatotoxicidade induzido pelo extrato de $C$. jamacaru, apesar de nenhuma evidência adversa tenha sido observada durante o tratamento com animais.

É importante lembrar que várias aminas biogênicas como a serotonina, a histamina e a tiramina desempenham importantes funções fisiológicas no homem e em outros animais, atuando como hormônios no sistema nervoso e nos processos de digestão e de síntese protéica. Devido à integração das aminas biogênicas no metabolismo humano, existem mecanismos para seu controle e degradação; esta última envolve diferentes rotas como desaminação oxidativa com monoamina oxidases (Ten Brink et al., 1990; Peters \& Kunz, 1995; Oliveira et al., 2004).

A tiramina, $N$-metiltiramina e hordenina são aminas bioativas que quando ingeridas em grandes quantidades podem provocar efeitos tóxicos, como as enxaquecas, crises hipertensivas e taquicardias, principalmente provocadas pela tiramina (Oliveira et al., 2004; Mosquera, 2005). Estes efeitos podem ser potencializados em indivíduos com comprometimento no sistema das monoaminaoxidases (MAO A e MAO B). Acredita-se que aminas farmacologicamente ativas de origem vegetal devam ser estudadas, uma vez que dependendo da quantidade presente podem vir a representar um risco à saúde devido à possibilidade de desencadear sintomas de intoxicação que podem inclusive comprometer diversos órgãos. $\mathrm{O}$ que provavelmente não é o caso de C. jamacaru, por não existir na literatura vigente relatos de intoxicação alimentar animal e/ou humana através de consumo in natura de constituintes do mandacaru, e nem de trabalhos que indiquem intoxicações por produtos derivados desse vegetal. Os achados bioquímicos encontrados nesta pesquisa podem sugerir retenção de alguns metabólitos biotransformados no fígado das ratas durante o terço médio da gestação. Assim, é necessário estudos futuros visando a continuidade deste efeito adverso, com outros parâmetros farmacológicos, inclusive com avaliação de uma possível relação do efeito hepatotóxico com as aminas bioativas presentes no extrato bruto de Cereus jamacaru DC.

\section{CONCLUSÃO}

Baseados nos resultados obtidos, conclui-se que a administração do extrato metanólico de Cereus jamacaru não produz efeitos tóxicos e nem alterações sobre a maioria dos parâmetros bioquímicos e hematológicos estudados em ratas Wistar grávidas. Entretanto, o aumento dos níveis séricos de AST e ALT após o tratamento com o referido extrato, em diferentes doses administradas, apontaram para uma possível sobrecarga hepática, a qual deverá ser investigada mais detalhadamente em um estudo futuro.

\section{REFERÊNCIAS}

Agra MF, Baracho GS, Basílio IJD, Nurit K, Coelho VP, Barbosa DA 2007. Sinopse da flora medicinal do cariri paraibano. Oecol Bras 11: 323-330.

Albuquerque UP, Andrade LHC 2002. Uso de recursos vegetais da caatinga: o caso do agreste do estado de Pernambuco (nordeste do Brasil). INCI 27: 336-346.

Almeida MM, Tavares DPSA, Rocha AS, Oliveira LSC, Silva FLH, Mota JC 2006. Cinética da produção do fermentado do fruto do mandacaru. Rev Bras Prod Agroind 8: 3542.

Andrade CTS, Marques JGW, Zappi DC 2006a. Utilização de cactáceas por sertanejos baianos. tipos conexivos para definir categorias utilitárias. Sitientibus Ser Ci Biol 6: 3-12.

Andrade CTS, Marques JGW, Zappi DC 2006b. Utilização medicinal de cactáceas por sertanejos baianos. Rev Bras Pl Med 8: 36-42.

Barbosa HP 1998. Tabela da composição de alimentos do Estado da Paraíba Setor Agropecuário. 2ed. FAPEP-UFPB.

Bruton LL, Lazo JS, Parker KL 2006. Goodman \& Gilman: as bases farmacológicas da terapêutica. 11ed. São Paulo: McGraw-Hill-Interamericana do Brasil.

Cavalcanti NB, Resende GM 2006. Consumo do mandacaru (Cereus jamacaru P. DC) por caprinos na época da seca no semi-árido de Pernambuco. Caatinga 19: 402-408.

Cavalcanti NB, Resende GM 2007. Efeito de diferentes substratos no desenvolvimento de mandacaru (Cereus jamacaru P. DC.), facheiro (Pilosocereus pachycladus Ritter), xiquexique (Pilosocereus gounellei (A. Webwr Ex K. Schum.) Bly. Ex Rowl.) e coroa-de-frade (Melocactus bahiensis Britton \& Rose). Caatinga 20: 28-35.

Correa MP 1969. Dicionário das plantas úteis do Brasil, v.4. Rio de Janeiro: Imprensa Nacional.

Cubas ZS, Silva JCR, Catão-Dias JL (ed.) 2007. Tratado de animais selvagens medicina veterinária. São Paulo: Roca. Seção 5: p.432-474.

Dantas JA, Ambiel CR, Cuman RKN, Baroni S, Bersani-Amado CA 2006. Valores de referência de alguns parâmetros fisiológicos de ratos do Biotério Central da Universidade Estadual de Maringá, Estado do Paraná. Acta Sci Health Sci 28: 165-170.

Davet A, Carvalho JLS, Dadalt RC, Virtuoso S, Dias JFGD, Miguel MD, Miguel OG 2009. Cereus jamacaru: a non buffered LC quantification method to nitrogen compounds. Chromatographia 69: 245-247.

Ferro MM 2007. Caracterização neuroquímica e comportamental da lesão da via nigroestriatal com 1-metil-4-fenil-1,2,3,6- 
tetraidropiridina (mptp) e 6-hidroxidopamina (6-ohda) em ratos. Curitiba 66p. Tese de Doutorado, Programa de Pós-graduação em Ciências, Bioquímica, Universidade Federal do Paraná.

Gomes RP 1972. Fruticultura brasileira. São Paulo: Nobel.

Harkness SE, Wagner JE 1993. Biologia e clínica de coelhos e roedores. 3ed. São Paulo: Roca.

Jain NC 1993. Essentials of veterinary hematology. Philadelphia: Lea \& Febiger.

Lima JLS 1996. Plantas forrageiras das caatingas - uso e potencialidades, EMBRAPA CPATSA/PNE/RBG-KEW.

Magalhães A 2006. Perfil etnobotânico e conservacionista das comunidades do entorno da reserva natural Serra das almas, Ceará-Piauí, Brasil. Fortaleza, 68p. Dissertação de Mestrado, Programa Regional de Pós-graduação em Desenvolvimento e Meio Ambiente, Universidade Federal do Ceará.

Mann J 1987. Secondary metabolism. 2th ed. Oxford: Clarendon Press.

Milinković-tur S, Perić V, Stojević Z, Zdelar-Tuk M, Piršljin J 2005. Concentrations of total proteins and albumins, and AST, ALT and GGT activities in the blood plasma of mares during pregnancy and early lactation. Veterinarski arhiv 75: 195-202.

Moncorvo MCR, Silva CF, Santos STA, El-Warrak AO, Sebalhos S 1998. Tratamento homeopático da hepatotoxicose aguda induzida por tetracloreto de carbono em coelhos. Cien Rural 28: 405-409.

Mosquera JT 2005. Yajé y hongos alucinógenos, aspectos relacionados com su toxicidad. Av Enferm 239: 92-102.

Motta VT 2003. Bioquímica Clínica para o Laboratório: principios e interpretações. 4ed. São Paulo: Robe.

Oliveira HAC, Silva HCM, Sampaio AH, Viana FA, SakerSampaio S 2004. Determinação de histamina por cromatografia líquida de alta eficiência de fase reversa em atum e sardinha enlatados. Rev Cienc Agron 35:179188.

Peters N, Kunz B 1995. Microbial building and degradation of biogenic amines. Mededelingen Faculteit Landbouwwetenschappen Rijksuniversiteit Gent., 60: 1853-1860.

Pimentel Gomes R 2007. Fruticultura brasileira, 13ed. São Paulo: Nobel.

Salema S 1966. Doces em caldas, velha tradição brasileira. Jornal do Commercio. Rio de Janeiro. In: Jangada Brasil. 5: http://www.jangadabrasil.com.br/abril56/cp56040b.htm, acesso em 12 de fevereiro de 2006.

Silva AS, Figueiredo RMF, Queiroz AJM, Lima EE 2005a. Avaliação da composição físico-químico da coroa-defrade. BioTerra 5: 1-8.

Silva EJR, Aguiar FJS, Gonçalves ES, Sousa IMV, Dimech GS, Fraga MCCA, Coelho MCOC, Wanderley AG 2005b. Avaliação do tratamento subcrônico com o extrato hidroalcoólico de Calendula officinalis L. sobre os parâmetros bioquímicos e hematológicos em ratas Wistar. Rev Bras Farmacogn 15: 88-93.

Silveira SMC, Ramos AFH, Souza IA, Guerra MO, Peters VM, Vieira VA 2006. Perfil hematológico de ratas prenhes tratadas com Cereus jamacaru. XXI Reunião Anual da FESBE. Águas de Lindóia-SP, Brasil.

Souza IA, Melo UBC, Lima MCA, Higino JS 2000. Atividade toxicológica do extrato bruto do Cereus jamacaru DC. IX Congresso da organização de farmacêuticos Ibero-
Latinoamericanos, v.1. Salvador-BA, Brasil.

Souza IA, Lima MCA, Melo UBC, Higino JS 2001. Antitumour properties of Cereus jamacaru on na experimental model of cancer in: fundamental \& Clinical Pharmacology. 3rd Meeting of the federation of the European Pharmacological Societies, v. 15. Lyon, France.

Swenson MJ 1988. Propriedades fisiológicas e constituintes celulares e químicos do sangue. In:___. Fisiologia dos animais domésticos. 10 ed., Rio de Janeiro: Guanabara Koogan.

Ten Brink B, Damink C, Joosten HMLJ, Huis In’t Veld JHJ 1990. Occurrence and formation of biologically active amines in foods. Int J Food Microbiol 11: 73-84.

Vieira SM 2006 Metodologia analitica para aminas bioativas como indice de autenticidade e da proporção de suco em refrigerante de laranja. 121p. Tese de Doutorado, Programa de Pós-graduação em Ciência de Alimentos, Universidade Federal de Minas Gerais.

Zappi D, Aona L 2007. Cactaceae in Flora brasiliensis revisitada. http://flora.cria.org.br, acesso em junho de 2007. 